\title{
Scale Anomaly as the Origin of Time
}

\author{
Julian Barbour ${ }^{1,2}$, Matteo Lostaglio ${ }^{3 *}$, and Flavio Mercati ${ }^{4,5, \dagger}$ \\ ${ }^{1}$ College Farm, South Newington, Banbury, Oxon, OX15 4JG, UK, \\ ${ }^{2}$ Department of Physics, Oxford University, Denys Wilkinson Building, Keble Road, OX1 3RH, UK, \\ ${ }^{3}$ Dep. of Physics, Imperial College London, Prince Consort Rd, London SW7 2BW, UK, \\ ${ }^{4}$ School of Mathematical Sciences, University of Nottingham, NG7 2RD, UK, \\ ${ }^{5}$ Perimeter Institute for Theoretical Physics, 31 Caroline Street North Waterloo, ON N2L 2Y5, Canada.
}

\begin{abstract}
We explore the problem of time in quantum gravity in a point-particle analogue model of scale-invariant gravity. If quantized after reduction to true degrees of freedom, it leads to a time-independent Schrödinger equation. As with the Wheeler-DeWitt equation, time disappears, and a frozen formalism that gives a static wavefunction on the space of possible shapes of the system is obtained. However, if one follows the Dirac procedure and quantizes by imposing constraints, the potential that ensures scale invariance gives rise to a conformal anomaly, and the scale invariance is broken. A behaviour closely analogous to renormalization-group (RG) flow results. The wavefunction acquires a dependence on the scale parameter of the RG flow. We interpret this as time evolution and obtain a novel solution of the problem of time in quantum gravity. We apply the general procedure to the three-body problem, showing how to fix a natural initial value condition, introducing the notion of complexity. We recover a time-dependent Schrödinger equation with a repulsive cosmological force in the 'late-time' physics and we analyse the role of the scale invariant Planck constant. We suggest that several mechanisms presented in this model could be exploited in more general contexts.
\end{abstract}

\footnotetext{
* This work has been submitted in partial fulfillment of the Master Degree in Physics at the University of Pavia.

†Electronic address: barbourj@physics.ox.ac.uk, matteo.lostaglio12@imperial.ac.uk, fmercati@perimeterinstitute.ca
} 


\section{Contents}

I. Introduction

II. The Analogue Model

III. Quantization: Before or After Reduction?

IV. Quantization of the Analogue Model $\quad 7$
A. Eliminating the momentum and angular momentum constraints
$\begin{array}{ll}\text { B. Quantization of the Hamiltonian constraint } & 7\end{array}$
C. Separation of the problem $\quad 8$
D. The anomaly 9
$\begin{array}{ll}\text { E. Self-adjoint extensions } & 11\end{array}$

V. Renormalization Group Time $\quad 13$

A. The Callan-Symanzik equation 13

B. Renormalization-group flows 16

$\begin{array}{ll}\text { VI. Semiclassical limit } & 17\end{array}$

$\begin{array}{ll}\text { VII. The Three-Body Model } & 19\end{array}$

$\begin{array}{ll}\text { A. Phase-space reduction } & 19\end{array}$

B. The spectrum of the scale-invariant Hamiltonian $\quad 20$

C. The problem of the initial conditions in a scale-invariant model 22

$\begin{array}{ll}\text { VIII. Discussion and Conclusions } & 24\end{array}$

$\begin{array}{ll}\text { IX. Appendices } & 27\end{array}$

$\begin{array}{ll}\text { A. Jacobi coordinates } & 27\end{array}$

$\begin{array}{ll}\text { B. Diagonalization of the inertia tensor } & 27\end{array}$

$\begin{array}{ll}\text { Acknowledgements } & 28\end{array}$

$\begin{array}{lr}\text { References } & 29\end{array}$ 


\section{INTRODUCTION}

One important difficulty in canonical quantum gravity is the problem of time [1-3], which arises from the refoliation invariance of general relativity (GR). The Hamiltonian form of general relativity (GR) has a quadratic constraint at each space point and, at the classical level, many-fingered-time evolution. Canonical quantization in the case of a spatially closed universe, leads, at least formally, to the Wheeler-DeWitt equation, which does not contain time. This is the frozen-formalism problem. As yet, there is no agreed way in which our manifest experience of a flow of time can be matched to structure in the quantum theory. In addition, there are severe technical problems in implementing the Hamiltonian constraints by quantum operators.

In this paper, we shall not address these technical issues but will suggest that the frozenformalism problem might be solved simultaneously with a further, less often noted problem the failure of GR to be exactly scale invariant. This is best explained in terms of the GR initialvalue problem, for which the only robust and effective method of solution is York's based on foliation of spacetime by spacelike hypersurfaces of constant mean (extrinsic) curvature (CMC) [4-6]. Represented this way, GR is a theory in which conformal three-geometries interact with a single global variable, the rate at which space expands. This global degree of freedom, a last vestige of Newton's absolute space, is why GR is not exactly scale invariant. The failure is mysterious.

It is possible to construct an exactly scale-invariant theory of conformal three-geometries interacting with themselves alone - essentially GR without the global variable [7]. There also exists a closely analogous point-particle model of that theory [8]. Its key feature is replacement of the standard Newtonian potential by one homogeneous of degree -2 , which ensures scale invariance. Newtonian gravitational forces are exactly recovered but with additional attractive forces that ensure scale invariance but are vanishingly small except on cosmological scales.

The observation on which this paper is based is that Dirac-style quantization of the theory with this potential leads to a conformal anomaly. This breaks the scale invariance and leads to a renormalization-group ( $R G)$ flow governed by a dimensionsless scale parameter. We show that this leads simultaneously to 'time evolution' governed by the RG scale parameter. ${ }^{1}$ We call this double emergence and put it forward as a possible resolution of two fundamental problems: the frozen formalism and the lack of exact scale invariance in gravity. ${ }^{2}$

\footnotetext{
${ }^{1}$ The idea of considering RG as the origin of time in Shape Dynamics is due to S. Gryb. See also [9].

${ }^{2}$ Its predictions can also be compared with the model explored in [10,11] with Newtonian (degree -1) potential in which the single global degree of freedom that breaks scale invariance is traded classically for an internal time, after which the remaining dynamical degrees of freedom are all scale invariant. There are in fact two ways to achieve
} 
In this paper we give a self-contained treatment of the classical particle model with the degree -2 potential of ref. [8] and its quantization with double emergence of the RG scale and an effective time through breaking of the scale invariance. We also treat the three-body problem, which gives us an opportunity to introduce what appears to be an interesting distinguished initial condition 'at the origin of RG time'. We also explore a further novel feature of the quantization: it has dimensionless Planck constant.

A word about concepts. In the Newtonian three-body problem all $3 \times 3$ particle coordinates are classical and quantum observables. But if the three particles are the entire universe, the position, orientation, and size of the triangle they form cease to have any observable meaning. There is also no external clock to measure time. Only the possible shapes of the triangle remain as observables. The collection of all possible shapes is shape space $\mathcal{S}$. It is our fundamental concept. We insist that only functions defined on $\mathcal{S}$ are classical or quantum observables. Position, orientation, and size are all gauge. But we shall see that relative size, without becoming an observable, can play the role of an independent variable like time.

\section{THE ANALOGUE MODEL}

General relativity in the ADM formulation is a reparametrization-invariant theory, so its Hamiltonian vanishes on the solutions of the equations of motion. The particle model of [8] has a similar dynamical structure to GR but on a different phase space: $\mathbb{R}^{6 N}$, the space of the Cartesian coordinates of $N$ point particles $\mathbf{r}^{J}=\left(r_{1}{ }^{J}, r_{2}{ }^{J}, r_{3}{ }^{J}\right)$, or, interchangeably, $\mathbf{r}^{J}=\left(r_{x}{ }^{J}, r_{y}{ }^{J}, r_{z}{ }^{J}\right)$, together with their conjugate momenta $\mathbf{p}^{J}=\left(p_{1}{ }^{J}, p_{2}{ }^{J}, p_{3}{ }^{J}\right)=\left(p_{x}{ }^{J}, p_{y}{ }^{J}, p_{z}{ }^{J}\right)$, where $J=1, \ldots, N$. The reduced phase space is defined by the algebra of constraints

$$
H=\sum_{J=1}^{N} \frac{\mathbf{p}^{J} \cdot \mathbf{p}^{J}}{2 m_{J}}+V=0,
$$

which implements reparametrization invariance, and

$$
\mathbf{P}=\sum_{J=1}^{\mathrm{N}} \mathbf{p}^{J}=0, \quad \mathbf{L}=\sum_{J=1}^{\mathrm{N}} \mathbf{r}^{J} \times \mathbf{p}^{J}=0,
$$

which implement translational and rotational invariance [12]. If $V$ is a function of the $N(N-1) / 2$ separations $\left\|\mathbf{r}^{J}-\mathbf{r}^{I}\right\|$ only, the algebra of constraints closes:

$$
\left\{L_{i}, L_{j}\right\}=\epsilon_{i j k} L_{k}, \quad\left\{L_{i}, P_{j}\right\}=\epsilon_{i j k} P_{k}, \quad\left\{P_{i}, P_{j}\right\}=0
$$

scale invariance, one (which we study here) stronger than the other (studied in $[10,11]$ ). 


$$
\left\{H, P_{i}\right\}=\sum_{J=1}^{N} \frac{\partial V}{\partial r_{i}^{J}}=0, \quad\left\{H, L_{i}\right\}=\sum_{J=1}^{N} \epsilon_{i j k} r_{j}^{J} \frac{\partial V}{\partial r_{i}^{J}}=0,
$$

where we used Einstein's summation convention and $\{\cdot, \cdot\}$ denotes the Poisson bracket

$$
\left\{r_{i}^{I}, p_{j}^{J}\right\}=\delta_{i j} \delta^{I J}
$$

This defines a theory of the evolution of relative configurations that can still differ in size. To implement scale invariance and define a theory on shape space, we add the constraint

$$
D=\sum_{J=1}^{N} \mathbf{r}^{J} \cdot \mathbf{p}^{J}
$$

Following [8] we call $D$ the dilational momentum. It has the same dimensions as the angular momentum $\mathbf{L}$ and measures expansion of the system just as $\mathbf{L}$ measures rotation. A scale-invariant theory clearly cannot allow physical expansion, for that requires an absolute scale. Vanishing of (6) is a necessary condition for scale invariance. But it is not sufficient: (6) imposes further constraints on $V$. Indeed, the Poisson algebra now also includes

$$
\left\{D, P_{j}\right\}=-P_{j}, \quad\left\{D, L_{j}\right\}=0, \quad\{H, D\}=-2 H+\sum_{J=1}^{N} r_{j}^{J} \frac{\partial V}{\partial r_{j}^{J}}+2 V,
$$

and it closes if $\sum_{J=1}^{N} r_{j}^{J} \frac{\partial V}{\partial r_{j}^{J}}+2 V=0$. By Euler's theorem this requires $V$ to be homogeneous of degree -2 in the coordinates $r_{j}^{I}$. The potential with this property chosen in [8] is

$$
V=\frac{V_{\mathrm{New}}}{R}, \quad V_{\mathrm{New}}=-\sum_{I<J} \frac{m_{I} m_{J}}{\left\|\mathbf{r}^{I}-\mathbf{r}^{J}\right\|}, \quad R^{2}=\frac{1}{M^{2}} \sum_{I<J=1}^{N} m_{J} m_{I}\left\|\mathbf{r}^{I}-\mathbf{r}^{J}\right\|^{2},
$$

where $M$ is the total mass and $M R^{2}$ is the centre-of-mass moment of inertia. The classical mechanics of this model reproduces Newtonian gravity to high accuracy in small enough subsystems but with a long-range attractive cosmological force that prevents the universe from expanding [8]. Indeed $R$ is a constant of motion.

In this paper we will study the quantum mechanics of this model. It turns out to be rich in interesting features. We first discuss an old issue in the quantization of constrained systems.

\section{QUANTIZATION: BEFORE OR AFTER REDUCTION?}

There are at least two ways to build a relational quantum theory. At first glance it seems most natural to get rid of the coordinate redundancies immediately and to quantize directly the true degrees of freedom. The other possibility is to impose the constraints quantum mechanically: this 
is Dirac quantization [13]. The two procedures are in general inequivalent, so which is the correct one?

The motivation for quantization after reduction is that the physical degrees of freedom live in the reduced phase space and therefore all the remaining ones are redundant (gauge) and unphysical. However, there are severe mathematical difficulties, generally insuperable, associated with working with true degrees of freedom, and for this reason alone the redundant degrees are usually retained.

We have four reasons not related to mere computational convenience to do the same:

1. There are several examples in physics of anomalies, in which a classical symmetry is not preserved by the quantum theory and the consequences have been confirmed experimentally. Quantization is by no means a unique procedure, as already operator ordering issues show, and a single classical theory usually corresponds to the classical limit of several quantum theories. Ultimately the observations tell us which quantization procedure provides the theory that fits them better.

2. In gauge theories the interactions are local when expressed redundantly but most awkwardly nonlocal in true degrees of freedom.

3. The laws of transformation from one set of redundant coordinates to another are very simple. In fact, Cartesian coordinates, like many other examples of redundant coordinates associated with an underlying Lie group, are crucial in suggesting the form of the kinetic energy. The point is that there is no unique prescription for choosing true degrees of freedom and, accordingly, no indication of how much kinetic energy should be associated with their rates of change. In contrast, the Cartesian coordinates are distinguished and democratic: weighted with the respective particle masses, they provide an obvious measure of the kinetic energy that should go with each. The residual ambiguity that arises from time-dependent group transformations between Cartesian frames is eliminated by means of constraints. Moreover, this approach selects a preferred form of these constraints, whereas in general one could get classically equivalent but quantummechanically different theories simply by multiplying the original constraints by arbitrary regular functions. This ambiguity is resolved if one works with group- theoretically defined redundant coordinates, which are distinguished and select the usual form of the Hamiltonian constraint.

4. The reduction before quantization approach also runs into the problem of recovering a meaningful notion of evolution in reparametrization-invariant theories like GR. 


\section{QUANTIZATION OF THE ANALOGUE MODEL}

\section{A. Eliminating the momentum and angular momentum constraints}

It is easy to show that the two quantizations are equivalent for the momentum constraint $\boldsymbol{P}=$ 0 , which can be conveniently solved by moving to mass-weighted Jacobi coordinates $\left\{\boldsymbol{\rho}^{K}\right\}, K=$ $1, \ldots, N-1$. These are a relational $N$-body generalization of the reduced-mass coordinates used to decouple the centre-of-mass motion in the two-body problem. There are many choices of Jacobi coordinates for one and the same $N$-body system. We refer to the Appendix IX A for details.

Suppose we have chosen Jacobi coordinates $\left\{\boldsymbol{\rho}^{J}\right\}$. We denote by $\left\{\boldsymbol{\pi}^{J}\right\}, \boldsymbol{\pi}^{J}=\left(\pi_{1}^{J}, \pi_{2}{ }^{J}, \pi_{3}{ }^{J}\right)$, the momenta conjugate to $\left\{\boldsymbol{\rho}^{J}\right\}$. In the new coordinates, the moment of inertia is mapped into the barycentric moment of inertia [14]:

$$
R^{2}=\sum_{J=1}^{N-1} \rho^{J} \cdot \rho^{J}
$$

The new coordinates automatically implement the constraint $\mathbf{P}=0$, so we just need to consider the constraints $\mathbf{L}=0$ and $D=0$. The dilatational momentum constraint (6) becomes

$$
D=\sum_{J=1}^{N-1} \boldsymbol{\rho}^{J} \cdot \boldsymbol{\pi}^{J}=0
$$

and the Hamiltonian and angular momentum constraints are [3]

$$
H=\sum_{J=1}^{N-1} \frac{\boldsymbol{\pi}^{J} \cdot \boldsymbol{\pi}^{J}}{2}+\frac{V_{\mathrm{New}}}{R}=0, \quad \mathbf{L}=\sum_{J=1}^{\mathrm{N}} \boldsymbol{\rho}^{J} \times \boldsymbol{\pi}^{J}=0 .
$$

The constraint $\mathbf{L}=0$ can be solved by a gauge fixing (see Appendix IX B). ${ }^{3}$ However, our main focus is scale invariance. From now on, therefore, we will keep the rotational gauge unfixed and solve the constraint $\mathbf{L}=0$ by Dirac quantization. An exception will be the three-body problem, which is always planar when $\mathbf{L}=0$ and has a simple algebraic phase-space reduction.

\section{B. Quantization of the Hamiltonian constraint}

The Poisson brackets between $\rho^{J}$ and $\pi^{J}$ are

$$
\left\{\rho_{i}^{I}, \pi_{j}^{J}\right\}=\delta_{i j} \delta^{I J}
$$

\footnotetext{
${ }^{3}$ The $S O(3)$ gauge symmetry of $N$-body dynamics is probably responsible for a lot of interesting features of this model, in particular at the quantum level, as shown by the wealth of results obtained by Littlejohn and Reinsch [14], who took seriously the fibre-bundle structure of the configuration space.
} 
We quantize these relations by promoting $\rho_{i}^{I}$ and $\pi_{j}^{J}$ to operators $\hat{\rho}_{i}^{I}, \hat{\pi}_{j}^{J}$ that act on the Hilbert space $\mathscr{L}^{2}\left(\mathbb{R}^{3 N-3}\right)$ and close the Heisenberg algebra

$$
\left[\hat{\rho}_{i}^{I}, \hat{\pi}_{j}^{J}\right]=i \hbar_{\mathrm{si}} \delta_{i j} \delta^{I J}
$$

A caveat: $\hbar_{\mathrm{si}}$ is not the familiar Planck constant, but a dimensionless number $\left(\hat{\rho}_{i}^{I}\right.$ has the dimensions of a length, while $\hat{\pi}_{j}^{J}$ is a length ${ }^{-1}$ ). We will see in Sec. VI how may be related to the usual dimensionful Planck constant.

The quantum Hamiltonian constraint (11) is

$$
\hat{H} \psi=\left(-\frac{1}{2} \hbar_{\mathrm{si}}^{2} \Delta_{3 N-3}+\frac{V_{\mathrm{New}}}{R}\right) \psi=0,
$$

where $\Delta_{3 N-3}$ is the Laplacian in $3 N-3$-dimensional Euclidean space.

In polar coordinates $\left(\rho_{1}^{1}, \rho_{2}^{1}, \rho_{3}^{1}, \ldots, \rho_{1}^{N-1}, \rho_{2}^{N-1}, \rho_{3}^{N-1}\right) \rightarrow(R, \sigma), R \in \mathbb{R}^{+}, \sigma \in S^{3 N-4}$,

$$
\hat{H} \psi(R, \sigma)=\frac{1}{2}\left[-\hbar_{\mathrm{si}}^{2} \frac{\partial^{2}}{\partial R^{2}}-\hbar_{\mathrm{si}}^{2} \frac{3 N-4}{R} \frac{\partial}{\partial R}+\frac{1}{R^{2}}\left(-\hbar_{\mathrm{si}}^{2} \Delta_{S^{3 N-4}}+2 V_{\text {shape }}\right)\right] \psi(R, \sigma)=0,
$$

where $\Delta_{S^{3 N-4}}$ is the spherical Laplacian, and we call $V_{\text {shape }}=R V_{\mathrm{New}}$ the shape potential [8, 12]. ${ }^{4}$ Its very interesting properties are investigated in the companion paper [11].

The reparametrization invariance of a relational theory like ours leads to a time-independent Schrödinger equation (15), the analogue in particle systems of the timeless Wheeler-DeWitt equation in geometrodynamics.

\section{Separation of the problem}

The potential $V=V_{\mathrm{New}} / R$ allows us to separate the variables in Eq. (15) ${ }^{5}$

$$
\psi(R, q)=\sum_{n} \xi_{n}(R) \varphi_{n}(\sigma)
$$

Substitution of this ansatz in (15) gives, for each $n$, the eigenvalue equation

$$
\hat{H}_{\text {shape }} \varphi_{n}=\left(-\hbar_{\text {si }}^{2} \Delta_{S^{3 N-4}}+2 V_{\text {shape }}\right) \varphi_{n}=\lambda_{n} \varphi_{n}
$$

\footnotetext{
${ }^{4}$ Saari [15] calls $V_{\text {shape }}$ the configuration measure.

${ }^{5}$ We shall show that the functions $\varphi_{n}$ are countable, so we adopt this notation already.
} 


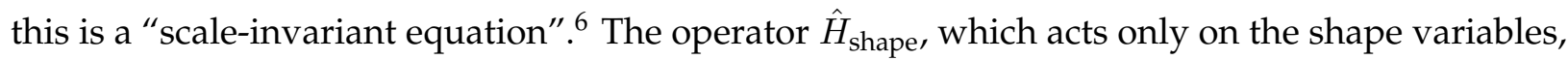
is self-adjoint on the dense domain $H^{2}\left(S^{3 N-4}\right.$ ) (Sobolev space of $\mathscr{L}^{2}$ functions whose derivatives up to the second order are $\mathscr{L}^{2}$ ). By the Weyl and Rellich-Kato theorems [17] and the compactness of $S^{3 N-4}$ it can be proved it has a bounded from below and discrete spectrum.

We still have to impose the constraints $\hat{L}_{j} \psi=0$ and $\hat{D} \psi=0$. The former acts only on the scale-invariant wavefunctions because it commutes with both $R$ and $\partial / \partial R$. $\hat{L}_{j}$ commutes also

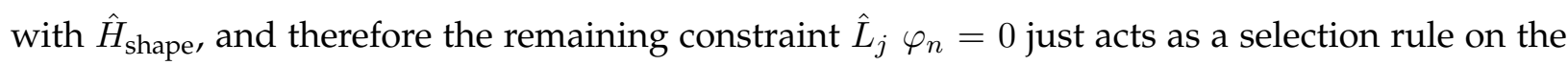
scale-invariant eigenfunctions $\varphi_{n}$. Applying this selection rule gives solutions defined on shape space, that is $\mathbb{R}^{3 N} / \operatorname{Similarity}\left(\mathbb{R}^{3}\right)$.

For each shape eigenvalue $\lambda_{n}$, we get a radial equation on $\mathscr{L}^{2}\left(\mathbb{R}^{+}\right)$:

$$
\left(-\hbar_{\mathrm{si}}^{2} \frac{\partial^{2}}{\partial R^{2}}-\hbar_{\mathrm{si}}^{2} \frac{3 N-4}{R} \frac{\partial}{\partial R}+\frac{\lambda_{n}}{R^{2}}\right) \xi_{n}=0,
$$

which can be nicely rewritten by means of the change of variables $u_{n}(R)=(\mu R)^{(3 N-4) / 2} \xi_{n}(R)$, where $\mu>0$ is an arbitrary scale with dimensions length ${ }^{-1}$, introduced to keep $u_{n}$ dimensionless:

$$
\hat{H}_{\mathrm{rad}}^{n} u_{n}=-\hbar_{\mathrm{si}}^{2} \frac{\partial^{2} u_{n}}{\partial R^{2}}+\frac{g_{n}}{R^{2}} u_{n}=0, \quad g_{n}=\lambda_{n}-\frac{1}{4}(3 N-4)(3 N-6) \hbar_{\mathrm{si}}^{2} .
$$

\section{The anomaly}

The anomalous behaviour of a $1 / R^{2}$ potential like the one in the radial equations Eq. (19) has been well-known since the pioneering work of Case [18] in the 1950s. Initially dismissed as nonphysical, its importance has only relatively recently been recognized in very different areas, from molecular physics to black holes (see e.g. [19] and the references therein). In particular there has been a surge of interest after the experimental confirmation [20,21] of the Efimov effect [22], which is now better understood as a consequence of this quantum anomaly.

The novelty of our approach is that we found, starting from relational first principles, similar equations describing a cosmological setting. A difference is that each radial equation is coupled with scale-invariant degrees of freedom and ultimately, through the anomaly, will determine their evolution in an emergent time. Let us develop the argument.

\footnotetext{
${ }^{6}$ One of the authors has proposed, in [16], that a notion of time might be hidden in the frozen wavefunction resulting from the reduction before quantization approach. This might be the case if the wavefunction of the universe is concentrated on 'time capsules', i.e., configurations which suggest a history. In the reduction-before-quantization approach, the only allowed solution of (17) is one with $\lambda_{n}=0$, corresponding to zero total energy and a frozen wavefunction. Rewriting (17) in the form $\hat{H}_{\text {shape }} \varphi_{n}=-\Delta_{S^{3 N-4}} \varphi_{n}+\left(2 / \hbar_{\mathrm{si}}^{2}\right) V_{\text {shape }} \varphi_{n}=\left(\lambda_{n} / \hbar_{\mathrm{si}}^{2}\right) \varphi_{n}$, we see that $2 / \hbar_{\mathrm{si}}^{2}$ plays the role of a dimensionless coupling constant. It might perhaps be possible to adjust its value to ensure the existence of the zero eigenvalue. This would be a novel first-principles derivation of the strength of quantum effects.
} 
We can see that a $1 / R^{2}$ potential can generate an anomaly in this way: consider the quantization of the dilatation constraint (6). There is a mild ordering ambiguity. Without loss of generality we will take the Weyl ordering

$$
\hat{D}=\frac{1}{2} \sum_{J, i}\left(\hat{\rho}_{i}^{J} \hat{\pi}_{i}^{J}+\hat{\pi}_{i}^{J} \hat{\rho}_{i}^{J}\right) \equiv-\frac{i}{2}\left(R \frac{\partial}{\partial R}+\frac{\partial}{\partial R} R\right) .
$$

From Eq. (7) and the canonical quantization we get

$$
[\hat{H}, \hat{D}] \psi=-2 \hat{H} \psi+i \hbar_{\mathrm{si}}\left(2 V+\sum_{J=1}^{N-1} \rho_{i}^{J} \frac{\partial V}{\partial \rho_{i}^{J}}\right) \psi:=-2 \hat{H} \psi+i \hbar_{\mathrm{si}} \hat{A} \psi .
$$

Classically, the quantity $\hat{A}$ vanishes for a $1 / R^{2}$ potential, due to Euler's theorem, but quantum mechanically this theorem breaks down, as we will see. The anomaly can be rewritten:

$$
\hat{A}=2 V+\underline{\rho} \cdot \nabla(V \cdot)=2 \frac{R^{3 N-5}}{2} \nabla \cdot\left(\frac{\underline{\rho} V}{R^{3 N-5}}\right),
$$

where $\underline{\rho}=\left(\rho_{1}^{1}, \ldots, \rho_{i}^{J}, \ldots, \rho_{3}^{N-1}\right)$ and $\nabla=\left(\partial / \partial \rho_{1}^{1}, \ldots, \partial / \partial \rho_{i}^{J}, \ldots, \partial / \partial \rho_{3}^{N-1}\right)$.

$$
\hat{A}=2 \frac{R^{3 N-5}}{2} \nabla \cdot\left(\frac{\rho}{R_{\text {shape }}^{3 N-3}}\right)=2 \frac{R^{3 N-5}}{2} \nabla \cdot\left(\frac{\underline{\rho}}{R^{3 N-3}}\right) V_{\text {shape }}+\frac{1}{R^{2}} \underline{\rho} \cdot \nabla V_{\text {shape }} .
$$

Defining $\Omega_{3 N-4}$ as the surface of a $3 N-4$ dimensional sphere, we have

$$
\nabla \cdot\left(\frac{\underline{\rho}}{R^{3 N-3}}\right)=\Omega_{3 N-4} \delta^{3 N-3}(\rho), \quad \hat{A}=R^{3 N-5} \Omega_{3 N-4} \delta^{3 N-3}(\underline{\rho}) V_{\text {shape }}+\frac{1}{R^{2}} \underline{\rho} \cdot \nabla V_{s i} .
$$

Equation (23) is exactly where the classical Euler theorem departs from the quantum one. Now $\delta^{3 N-3}(\underline{\rho})=\frac{1}{\Omega_{3 N-4} R^{3 N-4}} \delta(R)$, and because $V_{\text {shape }}$ does not depend on $R$ Euler's theorem holds for it. This implies

$$
\hat{A}=V_{\text {shape }} R^{-1} \delta(R)
$$

The distribution $A$ is called an anomaly. Our algebra does not close even if the potential is homogeneous of degree -2 ,

$$
[\hat{H}, \hat{D}]=-2 \hat{H}+i \hbar V_{\text {shape }} R^{-1} \delta(R)
$$

unless we ask for particular boundary conditions at the origin. We can interpret this result as a failure of Euler's theorem at the quantum level $[19,23]$. In the next section we will see that the closure of the algebra is incompatible with self-adjointness of the radial Hamiltonian. We should emphasize that self-adjointness is a working hypothesis that we retain in a theory of the whole universe, for which it is not a priori clear whether all the basic postulates of quantum theory, established in subsystems of the universe, are preserved. Caveat lector. 


\section{E. Self-adjoint extensions}

The radial equations (19) on the dense domain

$$
\mathscr{D}\left(H_{\text {rad }}^{n}\right)=\left\{u, u^{\prime} \text { absolutely continuous and } u(0)=u^{\prime}(0)=0\right\},
$$

have been studied extensively in the literature [19]. The deficiency indices depend on the energy regime. They are

1. $(0,0)$ if $g_{n} / \hbar_{\mathrm{si}}^{2}>3 / 4$, so that the radial Hamiltonian is self-adjoint on $\mathscr{L}^{2}\left(\mathbb{R}^{+}\right)$.

2. (1,1) if $-1 / 4<g_{n} / \hbar_{\mathrm{si}}^{2}<3 / 4$, so that there is a $U(1)$ group of self-adjoint extensions, labelled by the index $\theta$. Scale invariance is generally broken, but there are two conformal fixed points of the renormalization group (RG) flow. We will call this the weak coupling regime.

3. (1,1) if $g_{n} / \hbar_{\mathrm{si}}^{2}<-1 / 4$, but each element of the $U(1)$ group of self-adjoint extensions breaks scale invariance, i.e., there are no conformal fixed points in the RG flow. A limit cycle behaviour emerges. We will call this the strong coupling regime.

From the fundamental characterization of self-adjoint extensions [17], the domain of $\hat{H}_{\theta}^{n}$ is the direct sum of $\mathscr{D}\left(H_{\text {rad }}^{n}\right)$ and the span of

$$
w_{\theta}(\mathcal{R})=\sqrt{\mathcal{R}}\left[K_{\Xi_{n}}\left(e^{i \pi / 4} \mathcal{R}\right)+e^{-i \theta} K_{\Xi_{n}}\left(e^{-i \pi / 4} \mathcal{R}\right)\right], \quad \Xi_{n}=\sqrt{g_{n} / h_{s i}^{2}+1 / 4},
$$

consisting of a linear combination of the solutions of $\hat{H}_{r a d}^{n} u= \pm i \mu u$; we need $\mu$ for dimensional consistency; $\mathcal{R}=\mu R / \hbar_{\mathrm{si}}$, and $\Xi_{n}$ is the order of the Bessel function $K_{\Xi_{n}}$. The self-adjoint extensions break scale invariance, generating 'bound states' [19] with nonzero eigenvalues. With $\hat{H}_{\theta}^{n}$ denoting the $\theta$-extension of the radial operator $\hat{H}_{\text {rad }}^{n}$, the eigenstates are given by the equation

$$
\hat{H}_{\theta}^{n} u=-\kappa^{2} u \quad \Longrightarrow \quad-\frac{\partial^{2} u}{\partial \mathcal{R}^{2}}+\frac{g_{n}}{\hbar_{\mathrm{si}}^{2}} \frac{u}{\mathcal{R}^{2}}=-\frac{\kappa^{2}}{\mu^{2}} u
$$

The substitution $u(\mathcal{R})=\sqrt{\kappa \mathcal{R} / \mu} f(\mathcal{R})$ leads us to a modified Bessel equation for $f$; the two independent solutions are by definition

$$
u(\mathcal{R})=\sqrt{\frac{\kappa}{\mu} \mathcal{R}} K_{\Xi_{n}}\left(\frac{\kappa}{\mu} \mathcal{R}\right), \quad v(\mathcal{R})=\sqrt{\frac{\kappa}{\mu} \mathcal{R}} I_{\Xi_{n}}\left(\frac{\kappa}{\mu} \mathcal{R}\right),
$$

but, for both real and imaginary values of $\Xi_{n}$, only the first one is a solution in $\mathscr{L}^{2}(\mathbb{R})$. 
To choose a self-adjoint extension amounts to imposing a boundary condition at the origin in the previous problem, so we now want to understand which eigenfunctions $\sqrt{\kappa \mathcal{R} / \mu} K_{\Xi_{n}}\left(\frac{\kappa}{\mu} \mathcal{R}\right)$, corresponding to eigenvalues $-\kappa^{2}$, are compatible with the boundary condition

$$
\lim _{\mathcal{R} \rightarrow 0} u(\mathcal{R})=\lim _{\mathcal{R} \rightarrow 0} w_{\theta}(\mathcal{R})
$$

This condition gives a relation between $\kappa$ and $\theta$. Expanding $w_{\theta}$ and $u$ near the origin, we get

$$
\frac{\kappa_{n}^{2}}{\mu^{2}}=\exp \left[\frac{1}{\Xi_{n}} \log \left(\frac{\cos \left(\theta / 2-\pi \Xi_{n} / 4\right)}{\cos \left(\theta / 2+\pi \Xi_{n} / 4\right)}\right)\right] .
$$

The weak and strong coupling regimes have qualitatively different behaviours. In the weak coupling regime $\Xi_{n} \in[0,1]$ is real and Eq. (31) is one-to-one, so each self-adjoint extension selects one and only one state of the discrete spectrum. The function $\kappa_{n}(\theta)$ is defined only in the domain $\theta \in\left[0, \pi-\pi \Xi_{n} / 2\right] \bigcup\left(\pi+\pi \Xi_{n} / 2,2 \pi\right]$, where it is bijective (see Fig. 1, left-hand side). The two boundary points, $\pi-\pi \Xi_{n} / 2$ and $\pi+\pi \Xi_{n} / 2$, corresponding respectively to the values $\kappa=0$ and $\kappa=\infty$, merge at the transition $\Xi_{n} \rightarrow 0$ between the weak and strong coupling regimes (Fig. 1, right-hand side). This is relevant for the RG interpretation of the problem, as we will see later.

In the strong attractive regime $\Xi_{n}$ is imaginary, and this causes an abrupt change. The function $\kappa(\theta)$ is defined on the whole range $\theta \in[0,2 \pi)$, but is multivalued: in this case Eq. (31) can be rewritten as

$$
\frac{\kappa_{n m}^{2}}{\mu^{2}}=\exp \left[-\frac{\theta}{\left|\Xi_{n}\right|}+\frac{2}{\left|\Xi_{n}\right|} \arctan \left(\frac{\sin \theta}{\cos \theta+e^{\pi\left|\Xi_{n}\right| / 2}}\right) \pm \frac{2 \pi m}{\left|\Xi_{n}\right|}\right], \quad m \in \mathbb{N} .
$$

Each $\theta$ selects an entire discrete spectrum made of a tower of states with an accumulation point at zero. These are all the values of the energy compatible with the boundary condition fixed by
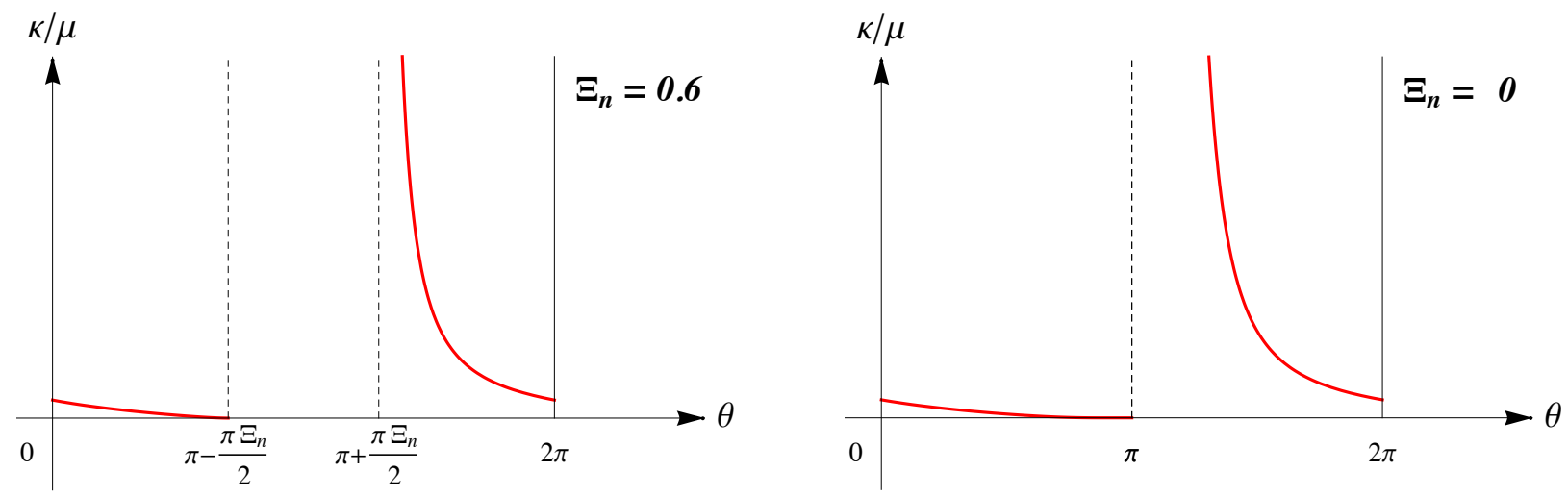

FIG. 1: $\kappa$ vs $\theta$ in the weak regime. The two preferred choices of $\theta$ correspond to $\kappa=0$ and $\kappa=\infty$. The boundary between the weak and strong regimes, where the two distinguished self-adjoint extensions merge, is at $\Xi_{n}=0$. 


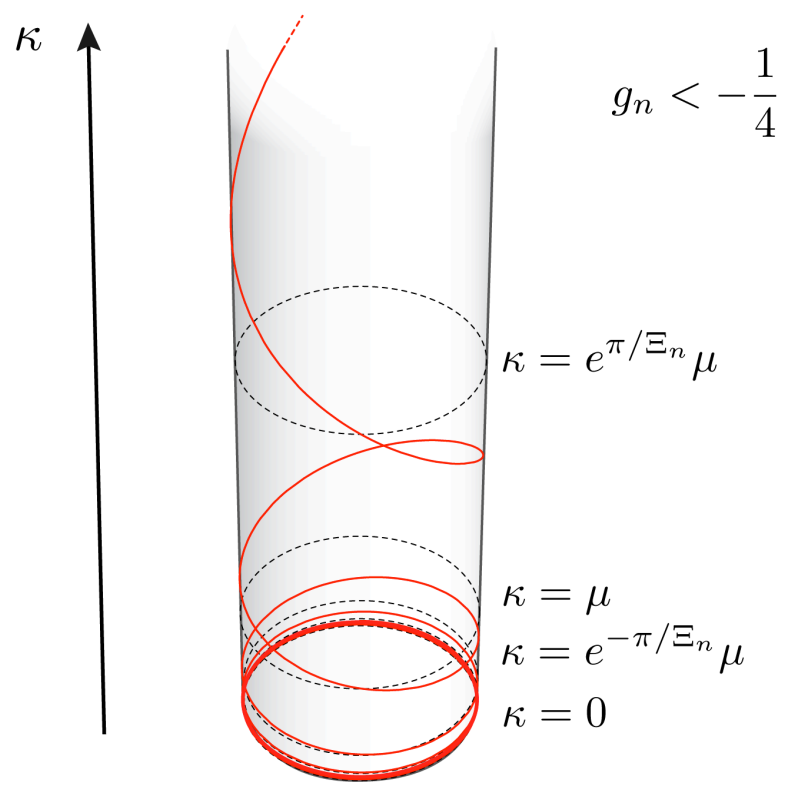

FIG. 2: $\kappa / \mu$ vs $\theta$ in a cylindrical representation in the strong regime. At each $\theta$ there is a conformal tower of states in a geometric sequence: the ratio between two neighbouring levels is a constant fixed by $\Xi_{n}$. Continuous variation of $\theta$ over a $2 \pi$ range, leads from one level of the tower to the next.

the chosen $\theta$. The motion of each level as $\theta$ is allowed to vary leads to a limit cycle behaviour, as explained in Fig. 2.

If we imagine the line twisting around the cylinder as a spring, a change in $\Xi_{n}$ corresponds to a change in its elongation. In fact the ratio between one level $E_{m}^{n}$ and the one immediately below, $E_{m-1}^{n}$, is equal to a constant that depends on $\Xi_{n}: E_{m}^{n} / E_{m-1}^{n}=e^{\pi /\left|\Xi_{n}\right|}$. This is known as the Efimov effect [22]; it is characterized by the presence of a 'conformal tower' of states in a geometric sequence $\left\{\ldots,\left(\frac{\kappa_{n}}{\mu}\right) e^{-2 \pi /\left|\Xi_{n}\right|},\left(\frac{\kappa_{n}}{\mu}\right) e^{-\pi /\left|\Xi_{n}\right|},\left(\frac{\kappa_{n}}{\mu}\right),\left(\frac{\kappa_{n}}{\mu}\right) e^{\pi /\left|\Xi_{n}\right|},\left(\frac{\kappa_{n}}{\mu}\right) e^{2 \pi /\left|\Xi_{n}\right|}, \ldots\right\}$.

\section{RENORMALIZATION GROUP TIME}

\section{A. The Callan-Symanzik equation}

The anomaly broke the constraint $\hat{D} \psi=0$, which does not need to be satisfied by the wavefunction anymore. But there is a domino effect involving the constraint $\hat{H} \psi=0$, which turns out to be incompatible with the requirement of self-adjointness of the radial Hamiltonian. ${ }^{7}$ We will

\footnotetext{
${ }^{7}$ This is just a consequence of the fact that the eigenvalue zero in the strong regime is an accumulation point in the spectrum of every self-adjoint extension of each radial Hamiltonian without being an eigenvalue associated with a
} 
shortly argue that this generates an RG flow.

The minimal ansatz that makes the theory consistent is to relax the Hamiltonian constraint by introducing an energy eigenvalue $-\kappa^{2}$,

$$
\hat{H} \psi=-\kappa^{2} \psi,
$$

which is not in conflict with the other constraints $\hat{\mathbf{P}} \psi=0$ and $\hat{\mathbf{L}} \psi=0$. Support for this minimal ansatz is that it maintains the reparametrization invariance of the classical theory, though no longer with energy eigenvalue $E=0$. Moreover from Eq. (31) this requirement fixes one and only one self-adjoint extension for each radial equation (19) at the subtraction point. They are the extensions $\bar{\theta}_{n}$ such that

$$
\kappa_{n m}\left(\bar{\theta}_{n}\right)=-\kappa^{2} \forall n .^{8}
$$

In other words, the single ansatz (33) not only makes the theory self-consistent but also fixes all the mathematical redundancies that arise from the theory of self-adjoint extensions.

Let us now study how the notion of time emerges in our model as an RG flow. The wavefunction $\psi$ splits into a sum in which the coefficients $u_{n}$ will be interpreted as couplings for the scale-invariant eigenfunctions $\varphi_{n}$,

$$
\psi(R, \sigma)=\sum_{n} u_{n}(R) R^{(4-3 N) / 2} \varphi_{n}(\sigma), \quad \sigma \in S^{3 N-4}
$$

the couplings $u_{n}$ flow differently under the RG flow, as detailed in the following subsection, in a way that is controlled solely by the scale-invariant energies $g_{n}$ with which each is associated. In other words, each scale-invariant eigenfunction determines its own RG evolution. Moreover, from (35) we see that an evolution of the $u_{n}$ 's amounts to a redistribution of the relative amplitudes of the scale-invariant eigenfunctions. This redistribution looks very similar to an evolution with respect to a 'time' that is the scale parameter of the RG flow. 9

This might appear rather formal, but the language of effective field theories provides us with a physical interpretation of what is going on. The Hamiltonian constraint implies Eq. (28) for the $u_{n}{ }^{\prime}$ s. This equation can be recast in an interesting form if we re-express it as an equation for the

proper eigenfunction.

${ }^{8}$ The label $m$ does not affect the choice of $\theta_{n}$ in the strong regime because it corresponds to a complete turn around the cylinder (2). Moreover in the weak regime the label $m$ is not present at all.

${ }^{9}$ An advantage of this proposal is that the usual internal time scenarios generically have great difficulty in meeting the requirement of monotonicity whereas in our framework time, being associated to RG flow, is monotonic by definition. 
logarithmic derivative of $u_{n}$ :

$$
\gamma_{n}(R)=\frac{R \frac{\partial u_{n}(R)}{\partial R}}{u_{n}(R)}, \quad u_{n}(R) \propto e^{\int_{0}^{\log R} \gamma_{n}\left(R^{\prime}\right) d \log R^{\prime}}
$$

The radial equation for $\gamma_{n}$ becomes

$$
\frac{\partial \gamma_{n}}{\partial \log R}=-\gamma_{n}^{2}+\gamma_{n}+\frac{g_{n}}{\hbar_{\mathrm{si}}^{2}}+R^{2} \kappa^{2}
$$

This last equation has the form of a Callan-Symanzik equation for a coupling $\gamma_{n}$ with betafunction

$$
\beta\left(\gamma_{n}, R\right)=\frac{\partial \gamma_{n}}{\partial \log R}=-\left(\gamma_{n}-1 / 2-\sqrt{\Xi_{n}^{2}+R^{2} \kappa^{2}}\right)\left(\gamma_{n}-1 / 2+\sqrt{\Xi_{n}^{2}+R^{2} \kappa^{2}}\right),
$$

Kaplan et al. [24] and Mueller et al. [25, 26] have already appreciated the equivalence of the Schrödinger equation for the $1 / R^{2}$ potential and the RG flow equations for a theory with this particular beta function. Following $[25,26]$, we provide an interpretation of the running of the couplings: Eq. (28) has a bad singularity at $R=0$ (a total collision, the 'big bang' of the model), and therefore one will seek to regularize the potential near the origin. ${ }^{10}$ For example one could take a a square-well cutoff [19]:

$$
V(R)=\left\{\begin{array}{ll}
g_{n} / R^{2}, & R>R_{0} \\
\gamma / R_{0}^{2}, & R \leq R_{0}
\end{array},\right.
$$

Then one has to match the solution and its derivative at the cutoff, and an efficient way to do this is to match the logarithmic derivatives at $R=R_{0}$ :

$$
\lim _{R \rightarrow R_{0}^{+}} \frac{R \frac{\partial \psi(R)}{\partial R}}{\psi(R)}=\lim _{R \rightarrow R_{0}^{-}} \frac{R \frac{\partial \psi(R)}{\partial R}}{\psi(R)} .
$$

This equation implies a relation between the coupling $\gamma$ and the cutoff radius $R_{0}$, which causes $\gamma$ to run. If we calculate the logarithmic derivative of the wavefunction inside the cutoff radius at $R_{0}$, we obtain $\gamma_{n}\left(R_{0}\right)$. As a function of $R_{0}, \gamma$ satisfies Eq. (38) [24]. The radial Schrödinger equation is equivalent to the RG group flow equations (37).

The usual interpretation in RG theory is that changing the cutoff corresponds to probing the system at different energies or, equivalently, at different scales. Actually the independent variable in an RG flow is not an energy but a ratio of energies. This matches the intuition that, if we are

\footnotetext{
${ }^{10}$ Regularizing the potential is a complementary approach to that of self-adjoint extensions because, like it, it amounts to fixing a boundary condition near the origin. But the self-adjoint extensions approach is non perturbative and regulator-independent, so we keep both languages at hand to supplement each other and guard ourselves against artefacts of the regularization choice.
} 
considering the entire universe, there is no scale external to it. In our cosmological model the RG flow parameter is a ratio of scales, and it gives rise to a running of the couplings $u_{n}$ 's that redistributes the probabilities among different scale-invariant eigenfunctions. The Universe at different (relative) scales will change, as the RG flow guides the probability from one region of shape space to another. ${ }^{11}$

\section{B. Renormalization-group flows}

Let us sketch the evolution of the couplings $u_{n}$ under the RG flow. This determines the relative weights of the wavefunctions in (35). There are three kinds of behaviour:

1. None of the $u_{n}$ 's corresponding to $g_{n}>3 / 4$ evolve. They are frozen at their initial values.

2. All the $u_{n}$ 's corresponding to $-1 / 4<g_{n}<3 / 4$ evolve under the RG flow between two fixed points of (38). One of them, which fixes $\kappa=0$, is a Friedrichs extension that effectively sets the anomaly to be zero. The other one corresponds to $\kappa=+\infty$. The $u_{n}$ 's have the following expressions:

$$
u_{n}(\mathcal{R}, \theta)=\sqrt{\frac{\kappa(\theta)}{\mu}} \mathcal{R} K_{\Xi_{n}}\left(\frac{\kappa(\theta)}{\mu} \mathcal{R}\right)=\sqrt{\frac{\kappa(\theta) R}{\hbar_{\mathrm{si}}}} K_{\Xi_{n}}\left(\frac{\kappa(\theta) R}{\hbar_{\mathrm{si}}}\right)
$$

Because the $u_{n}$ 's depend only on the product $\kappa(\theta) R$ it is clear that there is a duality between the evolution under the RG flow and the evolution of $R$, from the definition $\kappa(\theta) R=R(\theta)$. The radius of the universe, measured as a dimensionless ratio relative to the initial (subtraction) point, changes under the RG flow, renormalizing the couplings $u_{n}$ :

$$
u_{n}(R(\theta))=\sqrt{\frac{R(\theta)}{\hbar_{\mathrm{si}}}} K_{\Xi_{n}}\left(\frac{R(\theta)}{\hbar_{\mathrm{si}}}\right)
$$

The two fixed points are reached for $R=0$ and $R=\infty$. The infinite past and future are conformal: this is a realization of Strominger's proposal [27] that the evolution of the Universe results from the renormalization of a conformal field theory flowing between two fixed points, associated to dark-energy dominated epochs of accelerated expansion. ${ }^{12}$ The structure of this model, however, is conceptually richer: we also have a frozen regime and a cosmological Efimov effect.

\footnotetext{
${ }^{11}$ We will not discuss here the major interpretational issue - which as yet has not definitive answer - that every quantum theory of thel Universe must face: what is the meaning of these probabilities?

${ }^{12}$ See also the model proposed recently in [28] and related papers.
} 
3. If $g_{n}<-1 / 4$ we enter the Efimov effect region. The most general expression for each $u_{n}$ is a linear combination of all the eigenfunctions of the extended Hamiltonian:

$$
u_{n}(R(\theta))=\sum_{m} \sqrt{\kappa_{m}(\theta) \mathcal{R}} K_{\Xi_{n}}\left(\kappa_{m}(\theta) \mathcal{R}\right)
$$

We have the same correspondence between evolution under RG flow and evolution of $R$ as in the weak regime. Moreover, as we saw, the limit cycle behaviour implies that the following geometric relation holds: $\kappa_{m}=e^{m \pi / \Xi_{n}} \kappa$.

\section{SEMICLASSICAL LIMIT}

In this section we will study how the physics of our toy model appears at late times, far from $R=0$. In this limit we can regard $R$ as a "heavy" degree of freedom, in a way that we will make precise in the following discussion. ${ }^{13}$ Without loss of generality, let us rewrite the Hamiltonian constraint (33), substituting $\psi(R, \sigma)=\eta(R) \chi(R, \sigma)$. The evolution under RG flow is not unitary, so the function $\eta$ is fixed by the requirement that the function $\chi$ stays normalized on shape space, $\langle\chi, \chi\rangle=1$, where the scalar product is the shape Hilbert space $\mathscr{L}^{2}\left(S^{3 N-1}\right)$. Denoting with a prime the derivatives w.r.t. $R$,

$$
-\hbar_{\mathrm{si}}^{2}\left(\eta^{\prime \prime} \chi+2 \eta^{\prime} \chi^{\prime}+\chi^{\prime \prime} \eta\right)-\hbar_{\mathrm{si}}^{2} \frac{3 N-4}{R}\left(\eta^{\prime} \chi+\eta \chi^{\prime}\right)+\frac{\eta}{R^{2}}\left(-\hbar_{\mathrm{si}}^{2} \Delta_{S^{3 N-4}} \chi+V_{\text {shape }} \chi\right)=-\kappa^{2} \eta \chi .
$$

Denoting by $\langle\cdot, \cdot\rangle$ the scalar product on the scale-invariant Hilbert space $\mathscr{L}^{2}\left(S^{3 N-4}\right)$, and assuming that $\chi$ is normalized to 1 at each value of $R$, so that $\langle\chi, \chi\rangle=1$, the mean over the scale-invariant ("light") degrees of freedom of (44) gives

$$
\begin{gathered}
-\hbar_{\mathrm{si}}^{2} \eta^{\prime \prime}-2 \hbar_{\mathrm{si}}^{2} \eta^{\prime}\left\langle\chi, \chi^{\prime}\right\rangle-\hbar_{\mathrm{si}}^{2} \eta\left\langle\chi, \chi^{\prime \prime}\right\rangle-\hbar_{\mathrm{si}}^{2} \frac{3 N-4}{R}\left(\eta^{\prime}+\eta\left\langle\chi, \chi^{\prime}\right\rangle\right)+ \\
\frac{\eta}{R^{2}}\left[-\hbar_{\mathrm{si}}^{2}\left\langle\chi, \Delta_{S^{3 N-4}} \chi\right\rangle+\left\langle\chi, V_{\text {shape }} \chi\right\rangle\right]=-\kappa^{2} \eta\langle\chi, \chi\rangle \equiv-\kappa^{2} \eta .
\end{gathered}
$$

Let us suppose now that the mean values over the light degrees are vanishingly small, apart from $\left\langle V_{\text {shape }}\right\rangle=\left\langle\chi, V_{\text {shape }} \chi\right\rangle$. This is very reasonable because of our assumption that the shape degrees of freedom are very rapidly varing w.r.t. $R$. We get

$$
-\hbar_{\mathrm{si}}^{2} \eta^{\prime \prime}-\hbar_{\mathrm{si}}^{2} \frac{3 N-4}{R} \eta^{\prime}+\frac{\left\langle V_{\text {shape }}\right\rangle}{R^{2}} \eta=-\kappa^{2} \eta
$$

\footnotetext{
${ }^{13}$ We do not have the "multiple choice problem" of usual semiclassical approaches because any function $f$ of $R$ and $D, f(R, D)$, is singled out by the anomaly. This is a significant advantage of the shape-dynamic approach.
} 
Now substitute again this equation in (44) to get

$$
-2 \hbar_{\mathrm{si}}^{2}\left(\frac{\eta^{\prime}}{\eta}\right) \chi^{\prime}-\hbar_{\mathrm{si}}^{2} \chi^{\prime \prime}-\hbar_{\mathrm{si}}^{2} \frac{3 N-4}{R} \chi^{\prime}+\frac{1}{R^{2}}\left(-\hbar_{\mathrm{si}}^{2} \Delta_{S^{3 N-4}} \chi+V_{\text {shape }} \chi-\left\langle V_{\text {shape }}\right\rangle \chi\right)=0 .
$$

Under the hypothesis that $\eta$ is a complex field, it can always be rewritten as $\eta(R)=$ $\alpha(R) e^{i S(R) / \hbar_{\mathrm{si}}{ }^{14}}$ We substitute this in (46) and require the real and imaginary parts to vanish separately,

$$
\begin{gathered}
-\hbar_{\mathrm{si}}^{2} \alpha^{\prime \prime}+\alpha S^{2}-\hbar_{\mathrm{si}} \frac{3 N-4}{R} \alpha^{\prime}+\frac{\left\langle V_{\text {shape }}\right\rangle}{R^{2}} \alpha+\kappa^{2} \alpha=0 \\
-2 \hbar_{\mathrm{si}} \frac{\alpha^{\prime}}{\alpha} S^{\prime}-\hbar_{\mathrm{si}} S^{\prime \prime}-\hbar_{\mathrm{si}} \frac{3 N-4}{R} S^{\prime}=0
\end{gathered}
$$

Let us examine at Eq. (48) in the classical limit defined compatibly with our previous assumptions as the case in which the amplitude $\alpha$ varies much less than the phase $S$. In this limit Eq. (48) is a Hamilton-Jacobi equation

$$
S^{2}=-\kappa^{2}-\frac{\left\langle V_{\text {shape }}\right\rangle}{R^{2}}
$$

From $S^{\prime}=d R / d t^{\mathrm{em}}$, the Hamilton-Jacobi equation gives us a definition of an emergent classical time; it has the dimensions of a surface,

$$
d t^{\mathrm{em}}=\frac{d R}{\kappa \sqrt{-1-\frac{\left\langle V_{\text {shape }}\right\rangle}{R^{2} \kappa^{2}}}} .
$$

Let us recast physics in this emergent classical time. Substituting (50) and (49) in (47)

$$
-2 i \hbar_{\mathrm{si}} S^{\prime} \chi^{\prime}+\hbar_{\mathrm{si}}^{2} \frac{S^{\prime \prime}}{S^{\prime}} \chi^{\prime}-\hbar_{\mathrm{si}}^{2} \chi^{\prime \prime}+\frac{1}{R^{2}}\left(-\hbar_{\mathrm{si}}^{2} \Delta_{S^{3 N-4}}+V_{\text {shape }}-\left\langle V_{\text {shape }}\right\rangle\right) \chi=0
$$

At late times we can disgregard the term $S^{\prime \prime} / S^{\prime} \propto 1 / R^{3}$. The term in $\chi^{\prime \prime}$ measures the kinetic energy associated to the overall expansion of the Universe. If this is neglibible w.r.t. the kinetic energy associated to the all whole of the shape degrees of freedom, we get the equation

$$
i \hbar_{\mathrm{si}} \frac{d \chi}{d t^{\mathrm{em}}}=-\frac{1}{R^{2}}\left(\hbar_{\mathrm{si}}^{2} \Delta_{S^{3 N-4}} \chi+\left(V_{\text {shape }}-\left\langle V_{\text {shape }}\right\rangle\right) \chi\right) .
$$

Because $R$ is a heavy degree of freedom, $\frac{1}{R^{2}} \Delta_{S^{3 N-4}} \chi \approx \Delta_{\mathbb{R}^{3 N-3}} \chi$, the previous equation finally becomes a time-dependent Schrödinger equation:

$$
i \hbar_{\mathrm{si}} \frac{d \chi}{d t^{\mathrm{em}}}=-\hbar_{\mathrm{si}}^{2} \Delta_{\mathbb{R}^{3 N-3}} \chi+\frac{1}{R} V_{\text {New }} \chi-\frac{\left\langle V_{\text {shape }}\right\rangle}{R^{2}} \chi .
$$

This equation is very interesting because it shows what could be achieved in a more sophisticated implementation of the ideas of this toy model:

\footnotetext{
${ }^{14}$ The assumption that $\eta$ is complex could be unjustified because is taken to be a solution of a real equation (see [29])
} 
1. It proves that our time-dependent Schrödinger equation could arise (for late-time physics, in a semiclassical limit) from a completely scale-invariant cosmological model as a consequence of the scale anomaly. A cosmological force appears. It is proportional to the mean value of the scale-invariant shape potential and repulsive.

2. If we ignore the cosmic origin of the dimensionful time (51) and consider (54) as the equation of an effective theory empirically determined, we would regard time as a fundamental unit and, for dimensional consistency, $\hbar_{\mathrm{si}}$ would be given the dimensions of an energy times a time. This is why we would write (54) with $\hbar$ instead of $\hbar_{\mathrm{si}}$, where $\hbar$ is considered a dimensionful constant. This suggests a general mechanism by which dimensionless fundamental constants in a scale-invariant model (in this case, $\hbar_{\mathrm{si}}$ ) can be replaced by dimensionful constants in a semiclassical approximation.

3. The fact that the 'gravitational constant' depends on the 'time' $R$ is a serious potential defect of the model that we do not wish to hide, especially since there are relatively strong bounds on the secular variation of Newton's constant. It should be remarked, however, that we derived Eq. (54) under the assumption that $R$ is almost constant compared with the observable degrees of freedom, so we cannot use it for predicting a cosmological evolution of $G$. Moreover, we are mainly interested in this toy model as a conceptual test ground for exploring the problem of time and scale invariance.

\section{THE THREE-BODY MODEL}

An important advantage of the three-body case is that the non-anomalous constraints $\hat{\mathbf{P}}=0$ and $\hat{\mathbf{L}}=0$ can be explicitly reduced before quantizing. But the reason why we are studying this problem is for the conceptual insights we will get from it. First we will get a physical understanding of what the scale-invariant Planck constant means. Secondly we will propose a natural solution to the question of setting initial-value conditions in our scale-invariant model. Both issues are interesting because seems they will not be restricted just to our particle toy-model.

\section{A. Phase-space reduction}

We will follow Montgomery [30] in the phase space reduction. The first simplification comes from the fact that, because the angular momentum $\mathbf{L}$ is zero, the problem is planar, so we can 
gauge fix two out of three angular momentum constraints plus one translational constraint by fixing the plane in which the motion stays. We are left with three two-component vectors $\mathbf{r}^{1}$, $\mathbf{r}^{2}$ and $\mathbf{r}^{3}$ that define the position of the three bodies on this plane. Then we gauge fix the two remaining translation constraints by going to mass-weighted Jacobi coordinates:

$$
\boldsymbol{\rho}^{1}=\sqrt{\frac{m_{1} m_{2}}{m_{1}+m_{2}}}\left(\mathbf{r}^{2}-\mathbf{r}^{1}\right), \quad \boldsymbol{\rho}^{2}=\sqrt{\frac{m_{3}\left(m_{1}+m_{2}\right)}{m_{1}+m_{2}+m_{3}}}\left(\mathbf{r}^{3}-\frac{m_{1} \mathbf{r}^{1}+m_{2} \mathbf{r}^{2}}{m_{1}+m_{2}}\right) .
$$

We are left with four coordinates $\rho^{1}, \rho^{2}$ and a single angular momentum constraint to gauge fix. The coordinates

$$
w_{1}=\frac{1}{2}\left(\left\|\boldsymbol{\rho}^{1}\right\|^{2}-\left\|\boldsymbol{\rho}^{2}\right\|^{2}\right), \quad w_{2}=\boldsymbol{\rho}^{1} \cdot \boldsymbol{\rho}^{2}, \quad w_{3}=\boldsymbol{\rho}^{1} \wedge \boldsymbol{\rho}^{2}
$$

are invariant under the remaining rotational symmetry and therefore give a complete coordinate system on the reduced configuration space. The norm of the vector $\mathbf{w}$ is proportional to the moment of inertia $\|\mathbf{w}\|^{2}=R^{2} / 4$, so the angular coordinates in the three-space $\left(w_{1}, w_{2}, w_{3}\right)$ coordinatize shape space, which has the topology of a sphere [30]. This shape sphere, as can be seen in Fig. 3, has several distinct regions of interest.

\section{B. The spectrum of the scale-invariant Hamiltonian}

For our purposes it suffices to say that the scale-invariant Hamiltonian takes the form [30] ${ }^{15}$

$$
\hat{H}_{\text {shape }}=-\hbar_{\mathrm{si}}^{2} \Delta_{S^{2}}+V_{\text {shape }},
$$

where $\Delta_{S^{2}}$ is the 2-dimensional spherical Laplacian and

$$
V_{\text {shape }}=-\sum_{I<J} \frac{\left(m_{I} m_{J}\right)^{3 / 2}}{\left(m_{I}+m_{J}\right)^{1 / 2}\left(m_{1}+m_{2}+m_{3}\right)^{2}} \frac{1}{\sqrt{1-\cos \chi \cos \left(\psi-\psi_{I J}\right)}},
$$

$\chi$ is the elevation angle from the equator and $\psi$ the azimuthal angle on the shape sphere. $\psi_{I J}$ is the azimuthal angle of the two-body collision between particles $I$ and $J$. Moreover, our Hilbert space will be $\mathscr{L}^{2}\left(S^{2}\right)$, with inner product

$$
(f, g)=\int_{S^{2}} d \sin \chi d \psi \bar{f}(\chi, \psi) g(\chi, \psi) .
$$

\footnotetext{
${ }^{15}$ Strictly speaking, the scale-invariant Hamiltonian is not self-adjoint due to the three singularities at the two-body collisions, and a $U(3)$ family of self-adjoint extensions would be needed. But these singularities are not as bad as the $1 / R^{2}$ singularity at the origin, because they go like $1 / R$. Moreover, these singularities reproduce the Newtonian gravitational interactions among particles, and there are good reasons just to take the Friedrichs extension for all of them, which are the extensions that allow us to best model the hydrogen atom.
} 


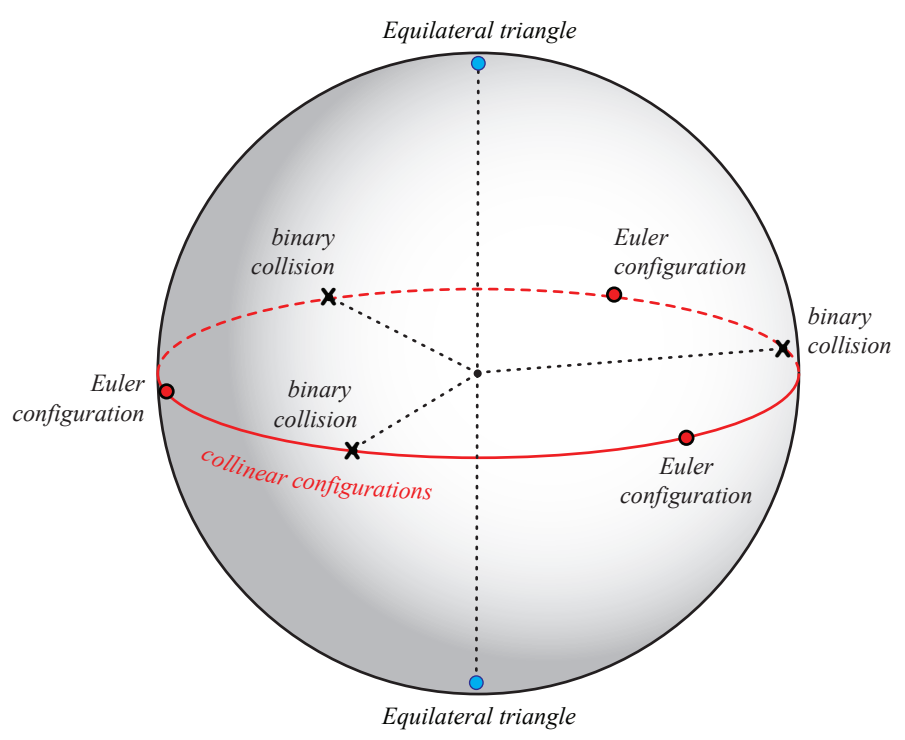

FIG. 3: The shape sphere of the three-body problem. The equator corresponds to the collinear configurations; at three points on it there are two-body collisions, where $V_{\text {shape }}$ is singular. Between them, at positions that depend on the mass ratios of the particles, there are saddle points of $V_{\text {shape }}$ (Euler configurations). The points in the southern hemisphere represent triangles which are mirror images of the corresponding ones (with the same longitude and opposite latitude) on the northern hemisphere. The north and south poles are equilateral triangles, at which, by a result due to Lagrange, $V_{\text {shape }}$ attains its absolute magnitude for arbitrary values of the masses. The critical points of $V_{\text {shape }}$ (Euler points and the equilateral triangles) are central configurations and play an important role in three-body theory. For example, if the system is 'held at rest' at one of them and then 'released', it will fall homothetically - without changing its shape - to a triple collision at the centre of mass, beyond which the solution cannot be continued. The figure is for the equal-mass case, with the binary collisions evenly spaced at $120^{\circ}$ intervals.

We will provide a qualitative description of the spectrum of $\hat{H}_{\text {shape }}$. We already mentioned that it can be proved that the spectrum is discrete and bounded from below. Let us start from the most excited states, with positive scale-invariant energy $\hat{H}_{\text {shape }} \varphi_{n}=\lambda_{n} \varphi_{n}$ and $\lambda_{n} \gg 1$. A convenient complete orthonormal basis in which to calculate the matrix elements of $\hat{H}_{\text {shape }}$ is that of the eigenfunctions of the spherical Laplacian, the spherical harmonics:

$$
-\Delta_{S^{2}} Y_{\ell}^{m}=\ell(\ell+1) Y_{\ell}^{m}
$$

It can be shown that in the expansion

$$
\left\langle Y_{\ell}^{m}\left|\hat{H}_{\text {shape }}\right| Y_{\ell^{\prime}}^{m^{\prime}}\right\rangle=\delta_{m m^{\prime}} \delta_{\ell \ell^{\prime}} \ell(\ell+1)+\int d \sin \chi d \psi \bar{Y}_{\ell}^{m} Y_{\ell^{\prime}}^{m^{\prime}} V_{s i}
$$

the matrix elements of $V_{\text {shape }}$ are bounded from above, being $V_{s i}$ bounded and harmonic functions normalized, while the term $\ell(\ell+1)$ grows arbitrarily large with $\ell$. Therefore asymptotically the 
spectrum is well approximated by that of the free particle on the sphere, that is

$$
\lambda_{n} \rightarrow \hbar_{\mathrm{si}}^{2} \ell(\ell+1), \text { for } n, \ell \text { sufficiently large. }
$$

For the less excited states, one gets a hyperfine splitting from the matrix elements $\left\langle Y_{\ell}^{m}\left|V_{\text {shape }}\right| Y_{\ell^{\prime}}^{m^{\prime}}\right\rangle$, which introduce an $m$-dependence of the eigenvalues.

Another regime in which we can say something qualitative about the spectrum is close to the ground state. In fact our problem is completely equivalent to that of a particle constrained on a sphere and subject to a potential that has three Coulombian wells. Close to one of the three singularities $\chi=0, \psi=\psi_{I J}$, the potential is well approximated by a Coulomb potential and the Laplacian is approximately flat:

$$
V_{\text {shape }} \sim \frac{\text { const. }}{\sqrt{\chi^{2}\left(\psi-\psi_{I J}\right)^{2}}}, \quad \Delta_{S^{2}} \sim \frac{\partial^{2}}{\partial \chi^{2}}+\frac{\partial^{2}}{\partial \psi^{2}}, \quad \chi, \psi \text { in a neighborhood of }\left(0, \psi_{I J}\right) .
$$

If $\hbar_{\mathrm{si}}$ is sufficiently small, the ground state and the first few excited states will resemble those of the hydrogen atom (or, rather, a linear combination of those of three independent hydrogen atoms). The reason why $\hbar_{\mathrm{si}}$ needs to be small enough is that $\hbar_{\text {si }}$ plays, for those wavefunctions, the role of a Bohr radius, setting a scale of quantum phenomena in shape space. Therefore it measures how far the wavefunction ventures from one of the singularities at $\left(0, \psi_{I J}\right)$. If it is localized enough, it will feel neither the curvature of the shape sphere nor the presence of the other singularities.

In the limit of small $\hbar_{\mathrm{si}}$ we are able to calculate the ground scale-invariant energy: it is (let us assume, for simplicity, that the three particles have the same mass)

$$
\lambda_{0} \sim-\frac{1}{4 \hbar_{\mathrm{si}}^{2}}
$$

Then from Eq. (19) we get

$$
g_{0} \sim-\left(\frac{1}{4}+\frac{1}{4}(3 N-4)(3 N-6)\right) \frac{1}{\hbar_{\mathrm{si}}^{2}}=-\frac{4}{\hbar_{\mathrm{si}}^{2}},
$$

which can be made smaller than $-1 / 4$ if $\hbar_{\mathrm{si}}$ is small enough. This means that some shape eigenstates enter the strong coupling regime.

\section{The problem of the initial conditions in a scale-invariant model}

We have, at this point, a qualitative picture of the features of our model. At least in the threebody case, we have established the form of the spectrum of the scale-invariant Hamiltonian, estimated the ground-state eigenvalue and determined its asymptotic behaviour for large eigenvalues. This spectrum is almost all that one needs to determine the $R$-evolution of the wavefunction. 
A further bit of information is needed, in the form of an overall normalization constant of each $u_{n}$ function. In fact choosing the self-adjoint extension (33) for all $n$ only fixes the (logarithmic) derivative of $u_{n}$ in Eq. (28), which we reproduce here for convenience,

$$
-\hbar_{\mathrm{si}}^{2} \frac{\partial^{2} u_{n}}{\partial R^{2}}+\frac{g_{n}}{R^{2}} u_{n}=-\kappa^{2} u_{n}, \quad g_{n}=\lambda_{n}-\frac{1}{4}(3 N-4)(3 N-6) \hbar_{\mathrm{si}}^{2} .
$$

This is a second-order differential equation and therefore depends on two initial conditions. The logarithmic derivative $u^{\prime} / u$ is fixed $\forall n$ by the self-adjoint extension via Eq. (30), and the other can be fixed by specifying an initial condition for the functions $u_{n}$, which can be set at a finite radius $R_{0}$

$$
u_{n}\left(R_{0}\right)=c_{n}, \quad c_{n} \in \mathbb{C},
$$

or at $R_{0}=0$ (through a limiting procedure), or even at $R_{0}=\infty{ }^{16}$

We propose now a way of fixing this initial condition; it relies on the highly asymmetric struc-

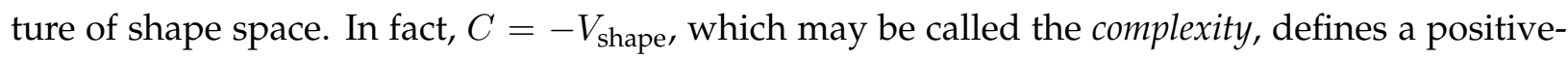
definite function on shape space that takes its minimum value on the configuration of the system that is more uniform than any other configuration. In the case of the three- and four-body problems for arbitrary values of the masses [31], the minimum of $C$ is at the equilateral triangle and regular tetrahedron respectively. Battye et al have done numerical calculations for up to $10^{4}$ particles which showed that the minimum of $C$ is realized on remarkably uniform (super-Poissonian) configurations [31].

The fact that all relative configuration spaces have a distinguished point was noted by one of us many years ago $[16,32,33]$ but before the importance of quotienting wrt dilatations as well as translations and rotations was fully appreciated. The distinguished point identified in [16, 32, 33] corresponds to the configuration with all particles collapsed to a single point and, for obvious reasons, was called Alpha. The additional quotienting wrt dilatations and the associated passage to shape space makes it possible to transform the qualitative intuition of the original idea into a quantitative theory because $C$ is an objective scale-invariant measure of complexity on shape space. It is a classical and quantum-mechanical observable. Simultaneously - and suggestively - it is, as we have shown, (minus) the potential that governs the scale-invariant dynamics.

We now define Alpha to be the most uniform shape of the system, where $C$ has its minimum. ${ }^{17}$

\footnotetext{
${ }^{16}$ We noted earlier, in footnote 6 , that in the case of quantization after reduction the spectrum, if it exists at all, consists solely of a zero eigenvalue. If $h_{\mathrm{si}}$ can be 'tweaked' to ensure existence of such an eigenvalue, the theory will still be incomplete if the eigenvalue is degenerate and corresponds to a superposition of zero-eigenvalue wavefunctions.

17 There is no corresponding notion of a most complex state: there is an Alpha, but no Omega [8, 11, 34].
} 
The presence of the Alpha point in shape space suggests the existence of a distinguished initial condition for the wavefunction of the universe: at early times it could be peaked around Alpha. When this choice is made, the expectation value of $V_{\text {shape }}$ is close to its maximum, the complexity close to a minimum.

\section{DISCUSSION AND CONCLUSIONS}

In this paper we have discussed the so-called problem of time in quantum gravity using an analogue particle model whose algebra of constraints is similar to the one of GR in Hamiltonian form. This model is almost uniquely fixed by relational first principles identical to those that one of us proved to be at the basis of GR $[12,35]$.

As we mentioned in the introduction, our basic idea is that two problems in GR could end up providing the solution to each other. We explored this possibility in the particle model. The two problems are the frozen nature of the Wheeler-DeWitt equation and the failure of GR in its usual representation ${ }^{18}$ to be fully relational: a single global degree of freedom, the volume of the Universe, cannot be considered as gauge. In this paper we insisted on strict classical relational principles by choosing a fully scale-invariant analogue model. We then discovered that time and scale emerge naturally through an anomaly that arises if we attempt a consistent Dirac canonical quantization.

We concluded that in Dirac quantization of the analogue model global scale invariance is an anomalous symmetry that is broken in the quantum theory. This is due to the singularity that the potential shows at a total collision. Its renormalization leads to an RG flow in which the radial eigenfunctions $u_{n}$ in the expansion

$$
\psi(R, \sigma)=\sum_{n} u_{n}(R) R^{(4-3 N) / 2} \varphi_{n}(\sigma), \quad \sigma \in S^{3 N-4}
$$

behave like RG running coupling constants for the shape eigenfunctions $\varphi_{n}$. In particular their logarithmic derivative satisfies a Callan-Symanzik equation. The behaviour of the couplings $u_{n}$ in Eq. (37) depends on the scale-invariant energy $g_{n}$ associated to $\varphi_{n}$ (see Eq. (17) and (19) for the definition of $g_{n}$ ). Above a certain limit, the couplings are frozen; then there is a regime in which they flow between two conformal fixed points; and finally the lowest energies exhibit an Efimov behaviour with limit cycles.

\footnotetext{
18 The caveat "in its usual representation" relates to the possibility noted in footnote 2 of achieving scale invariance by trading a global degree of freedom for an internal time.
} 
In this intriguing picture the dimensionless ratio of the scale of the universe relative to a reference value becomes 'time' in the sense that the changing scale of the universe renomalizes the couplings $u_{n}$, and this corresponds to a flux of probability on shape space. The scale ratio works this way because its change produces an RG flow that redistributes the relative probabilities among the shape eigenfunctions. We call this proposal double emergence, because scale and time emerge from the same quantum phenomenon, an anomalous symmetry breaking. ${ }^{19}$

What we have produced is a concrete implementation of the holographic cosmology scenario (albeit for a particle toy-model) that has very sound principles attached to it. In fact, our motivations have nothing to do with holography at all. This could be interesting from the point of view of finding a physical foundation of the AdS/CFT correspondence.

Besides this main result, in this paper we obtained a number of other interesting results and observations, which we summarize here.

1. The semiclassical regime. We studied the wavefunction of the system at large values of our 'time' $R$, under the assumption that the wavefunction fluctuates much more rapidly in the shape directions than in $R$ (Born-Oppenheimer approximation). With a WKB ansatz, we were able to find an effective time-dependent Schrödinger equation satisfied by the wavefunction, with a function of $R$ and $\left\langle V_{\text {shape }}\right\rangle$ playing the role of time. This effective semiclassical dynamics is expected to become dominant at late times (large $R$ 's). Our equation contains a repulsive cosmological force and a dynamically fixed Newton constant $G=1 / R$, but the equation is not valid if $R$ varies at cosmological scales.

2. The dimensionless Planck constant. The 'Planck constant' that appears in the canonical commutation relations of our model is a dimensionless number. This is due to the fact that the action is dimensionless, and the momenta have dimensions of inverse lengths. In the semiclassical, 'late-time' regime we were able to show a mechanism in which the familiar, dimensionful Planck constant could emerge. At the fundamental level time has no meaning, and the quantity that emerges at the classical level as time has the dimensions of a length squared. If one attributes to it a fundamental role, as is done when dealing with the effective physics that is relevant in the laboratory, then the Planck constant takes on some dimensions, which are needed to convert from squared lengths to times. This role of the Planck constant is identical to that of Boltzmann's constant in thermodynamics: fundamen-

\footnotetext{
${ }^{19}$ Admittedly this model is still affected by the quantum-mechanical measurement problem. The wavefunction in fact spreads over macroscopically distinguishable configurations in shape space.
} 
tally it is dimensionless, but at the effective level one attributes to it the dimensions that are necessary to convert from energy to heat, which at the fundamental (in that case, microscopical) level are the same thing. Even if eventually the particular toy model considered in this paper gets ruled out, we expect such a role for the Planck constant to emerge whenever one assumes that the fundamental degrees of freedom are dimensionless.

3. The three-body problem. We studied the case of three bodies, which is unique for the important simplifications that it allows: the angular momentum constraint can be algebraically solved, and shape space can be conveniently described as a two-dimensional sphere, whose radius is proportional to the moment of inertia $R$, our 'time'. The shape Hamiltonian is simply the spherical Laplacian on the shape sphere plus $V_{\text {shape, }}$ which is a relatively simple function on the sphere. A qualitative study of its spectrum was enough to establish that all of the three regimes of the RG flow are realized. Moreover the scale invariant Planck constant appeared as a scale of quantum phenomena on shape space.

4. The initial condition. The only thing that this model leaves unspecified is the initial condition for the wavefunction on shape space, which can be fixed by setting the coefficients $u_{n}$ at a certain value of $R$. Developing the classical proposal of a distinguished ' $\alpha$-point' in the configuration space of the scale-invariant degrees of freedom made in [34], we suggest that the driving potential of the scale-invariant dynamics, $V_{\text {shape, }}$ is also a natural measure of complexity in the system. In fact its maximum is attained the most uniform state, which we called the ' $\alpha$-state'.${ }^{20}$ So a natural initial condition for the wavefunction on shape space is such that all the probability is concentrated on this uniform shape, the equilateral triangle in the three-body problem. The evolution would then make the wavefunction spread out of that point, driving change in shape.

An interesting possibility is to extend these ideas to more general contexts. In particular it is intriguing to ask if GR could be considered as a fully conformal gauge theory on conformal superspace (the geometrodynamic counterpart of our shape space) in which a single global gauge degree of freedom becomes dynamical and a preferred notion of cosmological time emerges. The theory of shape dynamics [35-39] could be the natural context in which these ideas can be explored.

${ }^{20}$ See VIIC for a discussion which generalize this proposal to $\mathrm{N}$ body problems. 


\section{APPENDICES}

\section{A. Jacobi coordinates}

A simple algorithm [40] for creating Jacobi coordinates requires specification of a complete directed binary tree, whose leaves represent the $N$ particles, and the $N-1$ internal vertices are associated with the Jacobi coordinates. The algorithm works this way: given such an arbitrary tree, one assigns to each "parent" vertex four numbers: the three coordinates of the centre of mass of the two "child" vertices and the sum of their masses, which are taken of course from the leaves.

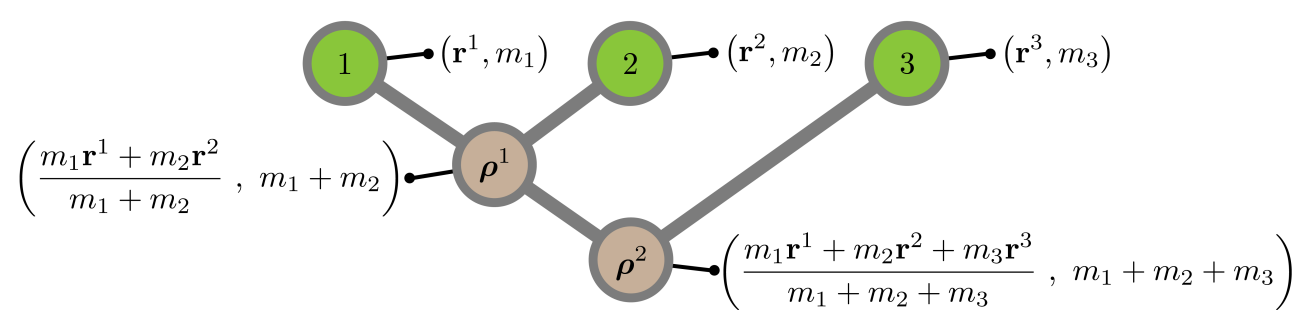

FIG. 4: The binary tree algorithm for the three-body system: the two Jacobi coordinates associated with the internal nodes are $\boldsymbol{\rho}^{1}=\mathbf{r}^{1}-\mathbf{r}^{2}, \boldsymbol{\rho}^{2}=\left(m_{1} \mathbf{r}^{1}+m_{2} \mathbf{r}^{2}\right) /\left(m_{1}+m_{2}\right)-\left(m_{1} \mathbf{r}^{1}+m_{2} \mathbf{r}^{2}+m_{3} \mathbf{r}^{3}\right) /\left(m_{1}+m_{2}+m_{3}\right)$.

Then one defines the Jacobi coordinates as a function on the internal nodes. This function associates to each node the difference of the coordinates of its two "child" nodes: $\rho^{\text {parent }}=\mathbf{r}^{\text {left }}$ $\mathbf{r}^{\text {right }}$. When applied to the root node, this function gives the $N$-th Jacobi coordinates, that is, the coordinates of the centre of mass of the system, which has now been decoupled and can be discarded, so that one can work with just with the first $3 N-3$ coordinates. The great advantage of the coordinates, besides the centre-of-mass decoupling, is that they leave the kinetic metric diagonal [40]. Its eigenvalues in this coordinate system are the Jacobi effective masses $\mu_{\mathrm{J}}$, which are just the masses of the nodes associated with each Jacobi coordinate. This algorithm represents a relational way to move to the centre-of-mass reference frame.

\section{B. Diagonalization of the inertia tensor}

We can solve the angular momentum constraint with a gauge-fixing, that is, with a choice of orientation of our axes. A global gauge-fixing, though, has to satisfy certain requirements to be good. The main requirement is that the constraint surface defined by the gauge fixing must never be parallel to the vector field generated by the constraint it is supposed to gauge fix. This translates into the requirement that the Poisson bracket between the gauge fixing and the constraint 
must be invertible, which means it must be everywhere (weakly) non-vanishing.

A popular choice of axes among $N$-body specialists is the one defined by the principal axes of the moment-of-inertia tensor [14]. It is attractive for its simplicity and symmetry, and more than anything else for its relational character, but it fails to be global for the reason mentioned above: it becomes degenerate at certain points of configuration space.

The moment-of-inertia tensor is defined as

$$
I^{a b}=\sum_{I=1}^{N} m_{I}\left(r_{a}^{I}-r_{a}^{\mathrm{cm}}\right)\left(r_{b}^{I}-r_{b}^{\mathrm{cm}}\right)-\delta^{a b} \sum_{I=1}^{N} m_{I}\left\|\mathbf{r}^{I}-\mathbf{r}^{\mathrm{cm}}\right\|^{2} .
$$

Its eigenvectors are the three principal axes of inertia of the $N$-body configuration. The matrix $I^{a b}$ is symmetric, and therefore can be diagonalized with a rotation that puts the principal axes of inertia respectively on the $x, y$ and $z$ axes. The three constraints that impose such a condition are the ones that set the off-diagonal elements to zero:

$$
I^{12}=I^{23}=I^{13}=0,
$$

This condition, though, does not always uniquely fix the axes for two reasons:

1. When the system is in a symmetric configuration (spherically or axisymmetric) there are identical eigenvalues (two if axisymmetric, three if spherically symmetric), and the matrix is already (block) diagonal.

2. Even in the non-symmetric case, the solution to equation (69) is not unique: there are three solutions, corresponding to the residual symmetry under exchange of the axes. But this is harmless as one can resolve the ambiguity by requiring the eigenvalues to be arranged in order of magnitude: $I^{11}<I^{22}<I^{33}$.

\section{Acknowledgements}

We would like to thank S. Gryb for his initial input that proved crucial for the beginning of this project. We thank also J. Louko, P. Hoehn and G. Canevari for useful comments and discussions during the preparation of this paper. M.L. thanks St. Hugh's College for hospitality when working on his Master Thesis in a joint exchange programme with Collegio Ghislieri; he also thanks the Institute for Advanced Studies of Pavia for partial funding.

This work was supported by a grant from the Foundational Questions Institute (FQXi) Fund, a donor advised fund of the Silicon Valley Community Foundation on the basis of proposal FQXi 
Time and Foundations 2010 to the Foundational Questions Institute. It was also made possible in part through the support of a grant from the John Templeton Foundation. The opinions expressed in this publication are those of the author and do not necessarily reflect the views of the John Templeton Foundation. Research at Perimeter Institute is supported by the Government of Canada through Industry Canada and by the Province of Ontario through the Ministry of Economic Development and Innovation.

[1] K. Kuchař, The problem of time in quantum geometrodynamics, pp. 169-195. Oxford University Press, New York, 1999.

[2] C. J. Isham, "Canonical quantum gravity and the problem of time," arXiv:gr-qc/9210011.

[3] E. Anderson, "The Problem of Time in Quantum Gravity," arXiv:1009.2157 [gr-qC] .

[4] J. J. W. York, “Gravitational degrees of freedom and the initial-value problem," Phys. Rev. Lett. 26 (1971) 1656-1658.

[5] J. J. W. York, "Role of conformal three geometry in the dynamics of gravitation," Phys. Rev. Lett. 28 (1972) 1082-1085.

[6] J. J. W. York, "Conformally invariant orthogonal decomposition of symmetric tensors on Riemannian manifolds and the initial value problem of general relativity," J. Math. Phys. 14 (1973) 456-464.

[7] E. Anderson, J. Barbour, B. Foster, and N. O’Murchadha, "Scale invariant gravity: Geometrodynamics," Class.Quant.Grav. 20 (2003) 1571, arXiv:gr-qc/0211022 [gr-qc].

[8] J. Barbour, "Scale-Invariant Gravity: Particle Dynamics," Class. Quant. Grav. 20 (2003) 1543-1570, arXiv:gr-qc/0211021.

[9] S. Gryb and F. Mercati, "Right about time?," arXiv:1301.1538 [gr-qc].

[10] J. B. Barbour, T. Koslowski, and F. Mercati, "The Solution to the Problem of Time in Shape Dynamics," (in preparation - provisional title) (2013) .

[11] J. B. Barbour, T. Koslowski, and F. Mercati, "Complexity and the Arrow of Time in Shape Dynamics," (in preparation - provisional title) (2013) .

[12] J. B. Barbour and B. Bertotti, "Mach's Principle and the Structure of Dynamical Theories," Proc. R. Soc. A 382 no. 1783, (1982) 295-306.

[13] P. A. M. Dirac, Lectures on Quantum Mechanics. Dover Publications, Yeshivea University, New York, 1964.

[14] R. Littlejohn and M. Reinsch, "Gauge fields in the separation of rotations and internal motions in the n-body problem," Rev. Mod. Phys. 69 (1997) 213.

[15] D. G. Saari, Collisions, Rings, and Other Newtonian N-Body Problems. American Mathematical Society, 2005.

[16] J. B. Barbour, "The Timelessness of quantum gravity. 2: The Appearance of dynamics in static 
configurations," Class. Quant. Grav. 11 (1994) 2875-2897.

[17] M. Reed and B. Simon, Methods of Modern Mathematical Physics, vol. 1: Functional Analysis. Academic press, 1972.

[18] K. Case, “Singular Potentials," Physical Review 20 (1950) 5.

[19] H. E. Camblong and C. R. Ordonez, "Anomaly in conformal quantum mechanics: From molecular physics to black holes," Phys.Rev. D68 (2003) 125013, arXiv: hep-th/ 0303166 [hep-th] .

[20] S. Inouye, M. R. Andrews, J. Stenger, H.-J. Miesner, D. M. Stamper-Kurn, and W. Ketterle, "Observation of Feshbach resonances in a Bose-Einstein condensate," Nature 392 (1998) 151-154.

[21] T. Kraemer, M. Mark, P. Waldburger, J. G. Danzl, C. Chin, B. Engeser, A. D. Lange, K. Pilch, A. Jaakkola, H.-C. Naegerl, and R. Grimm, “Evidence for Efimov quantum states in an ultracold gas of cesium atoms," Nature 440 (2006) 315-318, arXiv: cond-mat / $0512394 \mathrm{v} 2$.

[22] V. Efimov, "Energy levels arising from resonant two-body forces in a three-body system," Phys. Lett. B33 (1970) 563.

[23] G. N. Ananos, H. E. Camblong, C. Gorrichategui, E. Hernadez, and C. R. Ordonez, "Anomalous Commutator Algebra for Conformal Quantum Mechanics," Phys. Rev. D67 (2003) 045018, arXiv:hep-th/0205191v3.

[24] D. B. Kaplan, J.-W. Lee, D. T. Son, and M. A. Stephanov, “Conformality Lost,” Phys.Rev. D80 (2009) 125005, arXiv:0905.4752 [hep-th].

[25] Kolomeisky and Straley, "Renormalization-group analysis of the ground-state properties of dilute Bose systems in d spatial dimensions," Phys. Rev. B46 (1992) 12664.

[26] E. J. Mueller and T.-L. Ho, “Renormalization Group Limit Cycles in Quantum Mechanical Problems," arXiv:cond-mat/0403283.

[27] A. Strominger, "Inflation and the dS / CFT correspondence," JHEP 0111 (2001) 049, arXiv:hep-th/0110087 [hep-th].

[28] P. McFadden and K. Skenderis, "Holography for Cosmology," Phys. Rev. D81 (2010) 021301, arXiv:0907.5542 [hep-th].

[29] J. B. Barbour, “Time and complex numbers in canonical quantum gravity," Phys. Rev. D47 (1993) 5422-5429.

[30] R. Montgomery, “Infinitely Many Syzygies," Arch. Rational Mech. Anal. 164 no. 2002, 311-340.

[31] R. Battye, G. Gibbons, and P. Sutcliffe, "Central Configurations in Three Dimensions," Proc. R. Soc. A459 (2003) 911-943, arXiv: hep-th/ 0201101.

[32] J. Barbour and N. O'Murchadha, "Classical and quantum gravity on conformal superspace," arXiv:gr-qc/9911071.

[33] J. B. Barbour, "The Timelessness of quantum gravity. 1: The Evidence from the classical theory," Class. Quant. Grav. 11 (1994) 2853-2873.

[34] J. Barbour, "The end of time: The next revolution in physics,". Oxford University Press, UK.

[35] J. Barbour, "Shape Dynamics. An Introduction," arXiv:1105.0183. 
[36] H. Gomes, S. Gryb, and T. Koslowski, "Einstein gravity as a 3D conformally invariant theory," Class. Quant. Grav. 28 (2011) 045005, arXiv:1010.2481 [gr-qc] .

[37] H. Gomes and T. Koslowski, "The Link between General Relativity and Shape Dynamics," Class.Quant.Grav. 29 (2012) 075009, arXiv: 1101.5974 [gr-qc] .

[38] J. Barbour and N. O'Murchadha, “Conformal Superspace: the configuration space of general relativity," arXiv:1009.3559 [gr-qC].

[39] E. Anderson, J. Barbour, B. Z. Foster, B. Kelleher, and N. O’Murchadha, “The physical gravitational degrees of freedom," Class. Quant. Grav. 22 (2005) 1795-1802, arXiv: gr-qC/ 0407104.

[40] C. C. Lim, "Binary trees, symplectic matrices and the Jacobi coordinates of celestial mechanics," Archive for rational mechanics and analysis 115 no. 2, (1991) 153-165. 